$\underline{\text { Preprint typeset in JHEP style - PAPER VERSION }}$

arXiv:0706.1769

LPTHE-07

LPTENS-07-24

\title{
Higher Derivative Corrections, Dimensional Reduction and Ehlers Duality
}

\author{
Yann Michel ${ }^{\mathrm{a}}$ and Boris Pioline $\mathrm{a}^{\mathrm{a}, \mathrm{b}}$ \\ a Laboratoire de Physique Théorique et Hautes Energies*, \\ Université Pierre et Marie Curie - Paris 6, 4 place Jussieu, F-75252 Paris cedex 05 \\ ${ }^{b}$ Laboratoire de Physique Théorique de l'Ecole Normale Supérieure ${ }^{\dagger}$ \\ 24 rue Lhomond, F-75231 Paris cedex 05 \\ E-mail: ymichel@clipper.ens.fr, pioline@lpthe.jussieu.fr
}

\begin{abstract}
Motivated by applications to black hole physics and duality, we study the effect of higher derivative corrections on the dimensional reduction of four-dimensional Einstein, Einstein-Liouville and Einstein-Maxwell gravity to one direction, as appropriate for stationary, spherically symmetric solutions. We construct a field redefinition scheme such that the one-dimensional Lagrangian is corrected only by powers of first derivatives of the fields, eliminating spurious modes and providing a suitable starting point for quantization. We show that the Ehlers symmetry, broken by the leading $R^{2}$ corrections in Einstein-Liouville gravity, can be restored by including contributions of Taub-NUT instantons. Finally, we give a preliminary discussion of the duality between higher-derivative F-term corrections on the vector and hypermultiplet branches in $N=2$ supergravity in four dimensions.
\end{abstract}

\footnotetext{
${ }^{*}$ Unité mixte de recherche du CNRS UMR 7589

${ }^{\dagger}$ Unité mixte de recherche du CNRS UMR 8549
} 


\section{Contents}

1. Introduction and Summary $\quad 1$

2. Spherical Reduction and Higher-Derivative Terms 5

2.1 Pure Einstein Gravity 5

2.2 Einstein-Liouville Gravity 10

2.3 Einstein-Maxwell Gravity 12

3. Ehlers Symmetry Restored $\quad 15$

4. C-map with Higher Derivative Corrections 18

\section{Introduction and Summary}

In the study of stationary, spherically symmetric solutions to Einstein's gravity, possibly coupled to Maxwell and massless scalar fields, a useful trick is to first dimensionally reduce the action along the time-like Killing vector, and then only to enforce spherical symmetry of the three-dimensional spatial slices [1]. The advantage of this two-step procedure is that three-dimensional gauge fields arising in the reduction can be dualized after the first step into pseudo-scalars, leading to a non-linear sigma model with target space $\mathcal{M}_{3}^{*}$, coupled to three-dimensional gravity. The second step leads to a onedimensional dynamical system describing the geodesic motion of a fiducial particle on the cone $\mathbb{R}^{+} \ltimes \mathcal{M}_{3}^{*}$. The factor $\mathbb{R}^{+}$describes the radius of the two-sphere, while $\mathcal{M}_{3}^{*}$ is an analytic continuation of the moduli space $\mathcal{M}_{3}$ arising in the usual Kaluza-Klein reduction along a space-like direction, leading to a metric with indefinite signature. In many cases, $\mathcal{M}_{3}^{*}$ has a larger group $G_{3}$ of non-compact symmetries beyond those already manifest in four dimensions $\left(G_{4}\right)$, which may allow to integrate the geodesic motion explicitly.

The simplest examples where this procedure has been useful are SchwarzschildNUT black holes in pure Einstein gravity, which correspond to geodesics on the Poincaré upper half plane $S l(2, \mathbb{R}) / S O(2)[2]$. The $S l(2, \mathbb{R})$ action on this space, often known as Ehlers' symmetry, relates static solutions with solutions with non-zero NUT charge [2, 
3]. Similarly, Reissner-Nordström-NUT black holes in Einstein-Maxwell supergravity correspond to geodesics on $S U(2,1) / S l(2) \times U(1)[4,5]$ (see also the review [6] on this and other issues to be discussed below). The reduction of four-dimensional EinsteinMaxwell theories coupled to scalars valued in a symmetric space $G_{4} / K_{4}$ was worked out in $[1,7]$, leading to a non-linear sigma model on $G_{3} / K_{3}$, whose relation to $G_{4} / K_{4}$ can be most easily expressed using the language of Jordan algebras [8]. These theories are bosonic truncations of a special class of $\mathcal{N}=2$ supergravity theories with symmetric moduli spaces [9]. More generally, the reduction of $\mathcal{N}=2$ supergravity coupled to $n_{V}$ Abelian vector multiplets leads to a non-linear sigma model on a para-quaternionicKähler manifold $\mathcal{M}_{3}$ of dimension $4 n_{V}+4$, known as the $c^{*}$-map of the four-dimensional moduli space $\mathcal{M}_{4}[10,11,12]$. For a particular class of geodesics, corresponding to BPS black holes, the motion can be fully integrated (both classically and quantum mechanically) [13], and reproduces the usual attractor flow equations controlling the radial evolution of the scalars on $\mathcal{M}_{4}[12,6]$.

In all of these examples, the starting point was Einstein-Hilbert gravity in four dimensions coupled to abelian gauge fields and scalar fields, with canonical two-derivative kinetic terms for all fields. In general however, there are higher-derivative corrections to the four-dimensional effective action coming from integrating out massive modes in the full quantum theory, suppressed by inverse powers of the Planck mass $m_{P}$. A prime example are the $R^{2}$ corrections, which play an essential role in accounting for the microscopic entropy of "small black holes", whose horizon is singular in the twoderivative approximation: such corrections become dominant near the singularity, and lead to a smooth near-horizon geometry in agreement with thermodynamical expectations $[14,15,16,17,18,19,6]$. Higher derivative corrections are also important for regular black holes when trying to account for finite size corrections to the thermodynamical limit.

The main goal of this work is to analyze the effect of such higher derivative gravitational corrections at the level of the dimensional reduction from four space-time dimensions to one radial dimension. Our approach is also suitable for analyzing higherderivative corrections to the usual Kaluza-Klein reduction from four to three space-time dimensions, as we discuss further below. On general grounds, one expects higherderivative corrections to the geodesic motion on $\mathbb{R}^{+} \times \mathcal{M}_{3}$, preserving only the symmetries which originate from gauge and diffeomorphism invariance in four dimensions. As usual when working beyond two-derivative order, the exact form of the higher-derivative corrections is largely ambiguous due to the freedom of performing field redefinitions. A preferred frame is one in which only powers of first derivatives of the scalars appear, as it removes spurious modes found in other schemes $[16,17]$, and is amenable to canonical quantization by standard means. Moreover, it is also the natural frame in which to 
assess the existence of hidden symmetries, as will become clear shortly.

As the first part of our investigation, we consider pure Einstein gravity in four dimensions, with an arbitrary combination of the three curvature-squared invariants, and study its dimensional reduction on stationary spherically symmetric geometries. We show that all higher-derivative corrections in the one-dimensional Lagrangian can be removed by suitable field redefinitions. This could be anticipated from the fact that $R^{2}$ corrections in four dimensions can always be related to the Gauss-Bonnet density by an appropriate field redefinition of the four-dimensional graviton [20,32]. This is no longer true in the case of Einstein gravity coupled to a scalar field $\phi$, which we study next: allowing the coefficients of the $R^{2}$ terms to depend on $\phi$, we find that there exists a frame where only powers of first derivatives of the fields appear (with always at least one power of the first derivative of $\phi$, in agreement with the triviality of $R^{2}$ corrections with constant coefficients). Finally, we perform a similar analysis for Einstein gravity coupled to a single Maxwell field with general four-derivative couplings (but restricting for simplicity to the static case, rather than stationary). We find that higher-derivatives cannot in general be eliminated by field redefinitions, but that a first order scheme can still be found. We note that extremal solutions exist only when the coefficient of $\left(F_{\mu \nu}^{2}\right)^{2}$ vanishes, which may originate from the possibility of supersymmetrizing the higherderivative corrections.

As indicated above, our analysis also addresses the effect of higher-derivative corrections on the usual Kaluza-Klein reduction on a space-like direction, after analytic continuation of the scalar fields in the Maxwell directions. Of course, since we perform the reduction in the spherically symmetric sector only, the higher-derivative corrections in three-dimensions are only determined up to some tensor structure ambiguities.

In this context, an interesting question is whether higher-derivative interactions can be consistent with the extended non-compact symmetries $\left(G_{3}\right)$ that were present at tree-level, or at least with a discrete subgroup thereof. This question was raised long ago in the context of T-duality [21,22], where it was found that $\alpha^{\prime}$ corrections to the T-duality rules could be reabsorbed by field redefinitions, leaving an action invariant under $R \rightarrow 1 / R$. In the context of U-duality, the same question arises as to whether higher-derivative interactions, beyond the already well understood gravitational sector, preserve the duality symmetry $G_{3}(\mathbb{Z})=E_{8(8)}(\mathbb{Z})$ of M-theory compactified on a eighttorus [23, 24]. Since an $S l(2, \mathbb{R})$ subgroup of $G_{3}$ originates in the Ehlers symmetry of the reduction of four-dimensional Einstein gravity to three dimensions, we can investigate a toy version of this problem, and ask whether a discrete subgroup of the Ehlers symmetry can be preserved by $R^{2}$ corrections. For pure Einstein gravity, the answer to this question is trivial since such corrections can always be removed by field redefinitions. We therefore address this problem in the context of Einstein-Liouville theory, where 
such corrections are non-trivial, and break the Ehlers symmetry at order $\alpha^{\prime}$. Having found a frame where only powers of the first order derivatives of the scalar fields appear, it is straightforward to restore the invariance under a discrete $\operatorname{subgroup} S l(2, \mathbb{Z})$ of Ehlers symmetry: after upon expressing the Lagrangian in powers of the right-invariant form $p$ in $S l(2) / U(1)$ and its complex conjugate $\bar{p}$, with weight one under $U(1)$, it suffices to replace the coefficient of any term proportional to $p^{m} \bar{p}^{n}$ by a generalized Eisenstein series $f_{s, k=m-n}$ with $U(1)$ weight $k=m-n$, of the type considered in $[25,26]$. For $s=1$, the case relevant for $R^{2}$ corrections, $f_{s, k}$ can in fact be expressed in terms of ordinary almost holomorphic modular forms. The difference between the $S l(2, \mathbb{Z})$-invariant and the original dimensionally reduced Lagrangian can be attributed to Taub-NUT gravitational instantons, as well as loops of gravitons running along the compact circle. Thus, we give a precise realization of the general expectations expressed in $[23,24]$.

Finally, a third motivation for our work is to further our understanding of the duality between hypermultiplets and vector multiplets in three dimensions beyond the two-derivative level. In type II string theory compactified on a Calabi-Yau three-fold $Y$ times a circle, T-duality along the circle exchanges the hypermultiplet and vector multiplet moduli spaces of the type IIA and type IIB theories, respectively. Since the hypermultiplet sector is independent of the size of the circle, and since the metric for the three-dimensional vector multiplets is given by the $c$-map of the four-dimensional vector multiplet metric, this implies that, at tree-level, the four-dimensional hypermultiplet space is given by the $c$-map of the four-dimensional vector multiplet space of the dual theory $[10,11]$. The hypermultiplet metric is further corrected by D-instantons, dual to black holes winding around the circle on the vector-multiplet side. It is natural to guess that the same argument should relate the $R^{2} F^{2 h-2}$ "F-term" higher derivative corrections on the vector multiplet side in four dimensions to the $\left(\nabla^{2} S\right)^{2}(\nabla Z)^{2 h-2}$ " $\tilde{F}$ term" higher-derivative corrections on the hypermultiplet side. Here $(S, Z)$ denotes the two chiral fields of the universal hypermultiplet [27]. In Section 4, we give a preliminary analysis of this problem in the simplest case with $h=1$ and $\left(n_{V}, n_{H}\right)=(0,1)$, and conclude that the identication between $F_{1}$ and $\tilde{F}_{1}$ is more subtle than commonly thought.

The organization of this paper is as follows. In Section 2, we discuss the reduction of four-dimensional Einstein, Einstein-Liouville and Einstein-Maxwell gravity with fourderivative corrections to one radial dimension, and find suitable field redefinitions such that the resulting Lagrangian involves only powers of first derivatives of the fields. In Section 3, we discuss the restoration of Ehlers symmetry via instanton corrections. In Section 4, we give a preliminary discussion of the relation between the higherderivative F-term couplings $F_{1}$ and $\tilde{F}_{1}$ on the vector and hypermultiplet branch in 
three dimensions.

As this work was finalized, we received [31], which has some overlap with the results in Section 3. Note added: After the first version of this paper appeared on the archive, the authors of [32] pointed out that $R^{2}$ corrections in pure Einstein gravity can be completely removed by field redefinitions, not just in the sector with flat sections as we erroneously claimed. In this revised version, we extend our discussion to the case of Einstein-Liouville gravity, where $R^{2}$ corrections are non-trivial and do break the Ehlers symmetry.

\section{Spherical Reduction and Higher-Derivative Terms}

\subsection{Pure Einstein Gravity}

In this Section, we study pure gravity in four dimensions, with four-derivative corrections to the Einstein-Hilbert action:

$$
S=\int d^{4} x \sqrt{-g_{4}}\left[R^{(4)}+\alpha\left(\left[R_{\mu \nu}^{(4)}\right]^{2}-\left[R^{(4)}\right]^{2}\right)+\beta\left[R^{(4)}\right]^{2}+\gamma R_{G B}^{2}+o\left(\alpha^{\prime}\right)\right]
$$

and $o\left(\alpha^{\prime}\right)$ denotes further higher derivative corrections derivatives, which we assume to be negligible compared to the four-derivative interactions displayed in (2.1). Throughout this paper, we work perturbatively in $(\alpha, \beta, \gamma) \sim \alpha^{\prime}$. Since the Gauss-Bonnet density $R_{G B}^{2}=\left[R_{\mu \nu \rho \sigma}^{(4)}\right]^{2}-4\left[R_{\mu \nu}^{(4)}\right]^{2}+\left[R^{(4)}\right]^{2}$ is a total derivative in four dimensions, we set $\gamma=0$ in this section. The action (2.1) can then be specialized to the Riemann tensor squared $\left[R_{\mu \nu \rho \sigma}^{(4)}\right]^{2}$ or the Weyl tensor squared $\left[W_{\mu \nu \rho \sigma}^{(4)}\right]^{2}$ forms by setting $\alpha=3 \beta / 4$ or $\alpha=\beta / 6$, respectively.

In the presence of a time-like Killing vector, the four-dimensional metric may be written as

$$
d s_{4}^{2}=-e^{2 U}(d t+\omega)^{2}+e^{-2 U} d s_{3}^{2}
$$

where the scalar $U$, Kaluza-Klein one-form $\omega$ and spatial three-dimensional metric $d s_{3}^{2}$ are independent of the time coordinate $t$. The action (2.1) may then be reduced along the ansatz (2.2), leading to

$$
S_{3}=\int d^{3} x \sqrt{g_{3}}\left[R^{(3)}-2\left(\partial_{i} U\right)^{2}+\frac{1}{4} e^{2 U} F_{i j}^{2}+\mathcal{O}\left(\alpha^{\prime}\right)\right]
$$

where $F_{i j}=\partial_{i} \omega_{j}-\partial_{j} \omega_{i}$. The choice of powers of $e^{U}$ in (2.2) ensures that the threedimensional action is obtained in the Einstein frame. The terms $\mathcal{O}\left(\alpha^{\prime}\right)$ coming from the reduction of the four-derivative terms in (2.1) are somewhat cumbersome to obtain. For 
simplicity, and motivated by application to black holes, we further restrict to spherically symmetric solutions,

$$
d s_{4}^{2}=-e^{2 U}(d t+k \cos \theta d \phi)^{2}+e^{-2 U}\left[N^{2}(\rho) d \rho^{2}+r^{2}(\rho)\left(d \theta^{2}+\sin ^{2} \theta d \phi^{2}\right)\right]
$$

The integer $k$, often known as the NUT charge, describes the first Chern class of the Kaluza-Klein gauge field over the two-sphere at infinity; it can be dualized to a three-dimensional scalar $\sigma$ by adding a Lagrange multiplier $k \sigma^{\prime}$ to the action ${ }^{1}$, which ensures that $k$ is a constant of motion. It is worth emphasizing that the ansatz (2.4) follows entirely from the assumed isometries, in particular the Kaluza-Klein connection $k \cos \theta d \phi$ is unaffected by higher-derivative corrections to the Einstein-Hilbert action, and $\sigma^{\prime}$ will continue to be related to $k$ by Legendre transform. The lapse variable $N(\rho)$ can be viewed as an einbein along the radial direction $\rho$, and ensures that the resulting one-dimensional action is invariant under diffeomorphisms of $\rho$. For convenience we shall often set $N(\rho)=1$, the dependence on $N$ can be reinstated whenever needed by demanding reparametrization invariance.

It is now straightforward, if tedious, to compute the curvature invariants of the metric (2.4), and integrating over the sphere coordinates $\theta, \phi$ to obtain the one-dimensional Lagrangian. At two-derivative order, we find the familiar tree-level result

$$
\begin{aligned}
\mathcal{L}_{0} & =2 N\left[\left(\frac{r^{\prime}}{N}\right)^{2}-r^{2}\left(\frac{U^{\prime}}{N}\right)^{2}+\frac{e^{4 U}}{4 r^{2}} k^{2}+1\right]+k \sigma^{\prime} \\
& =\frac{2}{N}\left[r^{\prime 2}-r^{2}\left(U^{\prime 2}+\frac{1}{4} e^{-4 U} \sigma^{\prime 2}\right)+N^{2}\right]
\end{aligned}
$$

where in the second line we have performed the Legendre transform over the NUT charge $k$. The term in bracket is recognized as the metric on the cone $\mathbb{R}^{+} \times S l(2, \mathbb{R}) / U(1)$ where

$$
\tau=\sigma+i e^{2 U}
$$

is the standard coordinate on the upper half-plane. Stationary, spherically symmetric solutions of Einstein gravity in four dimensions are therefore described by the geodesic motion of a fiducial particle with unit mass on $\mathbb{R}^{+} \times S l(2, \mathbb{R}) / U(1)$. The $S l(2, \mathbb{R})$ symmetry acting on $\tau$ by fractional linear transformations

$$
\tau \rightarrow \frac{a \tau+b}{c \tau+d}
$$

is Ehlers's symmetry mentioned in the introduction.

\footnotetext{
${ }^{1}$ In the following the primes denote $\rho$-derivatives.
} 
Including the four-derivative interactions, and setting $N=1$, we arrive at $\mathcal{L}=$ $\mathcal{L}_{0}+\mathcal{L}_{1}$ where

$$
\begin{aligned}
\mathcal{L}_{1}= & (-2 \alpha+4 \beta) \frac{e^{2 U}}{r^{2}}+(8 \alpha-8 \beta) e^{2 U} U^{\prime 2}+(-4 \alpha+8 \beta) e^{2 U} U^{\prime \prime} \\
& +(4 \alpha-8 \beta) \frac{e^{2 U} r^{\prime 2}}{r^{2}}+(-8 \alpha+16 \beta) \frac{e^{2 U} r^{\prime} U^{\prime}}{r}+(12 \alpha-16 \beta) \frac{e^{2 U} r^{\prime \prime}}{r} \\
& +(-10 \alpha+16 \beta) e^{2 U} r^{\prime \prime 2}+(-2 \alpha+4 \beta) \frac{e^{2 U} r^{\prime 4}}{r^{2}}+(16 \alpha-32 \beta) e^{2 U} r^{\prime} r^{\prime \prime} U^{\prime} \\
& +(-8 \alpha+24 \beta) e^{2 U} r^{\prime 2} U^{\prime 2}+(8 \alpha-16 \beta) e^{2 U} r r^{\prime} U^{\prime 3}+(4 \alpha-8 \beta) e^{2 U} r^{2} U^{\prime 2} U^{\prime \prime} \\
& +(-12 \alpha+16 \beta) \frac{e^{2 U} r^{\prime 2} r^{\prime \prime}}{r}+(8 \alpha-16 \beta) e^{2 U} r r^{\prime \prime} U^{\prime \prime} \\
& +(4 \alpha-8 \beta) e^{2 U} r^{\prime 2} U^{\prime \prime}+16 \beta e^{2 U} r r^{\prime} U^{\prime} U^{\prime \prime} \\
& +(-8 \alpha+16 \beta) e^{2 U} r r^{\prime \prime} U^{\prime 2}+(8 \alpha-16 \beta) \frac{e^{2 U} r^{\prime 3} U^{\prime}}{r}+2 \beta e^{2 U} r^{2} U^{\prime 4}+4 \beta e^{2 U} r^{2} U^{\prime \prime 2} \\
& +(2 \alpha-4 \beta) k^{2} \frac{e^{6 U} r^{\prime \prime}}{r^{3}}+(2 \alpha+4 \beta) k^{2} \frac{e^{6 U} r^{\prime} U^{\prime}}{r^{3}}+(\alpha+2 \beta) k^{2} \frac{e^{6 U} U^{\prime \prime}}{r^{2}} \\
& +(2 \alpha-2 \beta) k^{2} \frac{e^{6 U} U^{\prime 2}}{r^{2}}+2 \beta k^{2} \frac{e^{6 U}}{r^{4}}-2 \beta k^{2} \frac{e^{6 U} r^{\prime 2}}{r^{4}}+\left(\frac{1}{2} \alpha+\frac{1}{4} \beta\right) k^{4} \frac{e^{10 U}}{r^{6}}
\end{aligned}
$$

It is worth noting that $\mathcal{L}_{0}$ and $\mathcal{L}_{1}$ are homogeneous, of degree 0 and -2 respectively, under the global symmetry

$$
\rho \rightarrow e^{2 l} \rho, \quad U(\rho) \rightarrow U(\rho)+l, \quad r(\rho) \rightarrow e^{2 l} r(\rho), \quad k \rightarrow k
$$

This reflects the homogeneity of the Einstein-Hilbert and $R^{2}$ terms, respectively, under global rescaling $g_{\mu \nu} \rightarrow e^{2 l} g_{\mu \nu}$.

The Lagrangian $\mathcal{L}$ should be supplemented by the Hamiltonian constraint, or Wheeler-De Witt equation, coming from the equation of motion of $N$. The latter can be reinstated by replacing all derivatives with respect to $\rho$ by covariant derivatives $\nabla_{\rho}$ with respect to the world-line metric $\gamma_{\rho \rho}=N^{2}$, contracted with appropriate powers of the inverse metric. This task is greatly simplified if one first performs field redefinitions and integration by parts such that the resulting action only involve powers of 
first order derivatives of $U(\rho)$ and $r(\rho)$. The most general redefinition ${ }^{2}$ is

$$
\begin{aligned}
\delta r= & \left(-\frac{5}{2} \alpha+4 \beta\right) e^{2 U} r^{\prime \prime}+(-\alpha+2 \beta) e^{2 U} r^{\prime} U^{\prime}+(2 \alpha-4 \beta) e^{2 U} r U^{\prime \prime} \\
& +\left(\frac{1}{4} \alpha-\beta\right) e^{2 U} r U^{\prime 2}+x_{1} \frac{e^{2 U}}{r}+x_{2} \frac{e^{2 U} r^{\prime 2}}{r}+x_{3} \frac{e^{6 U}}{r^{3}} k^{2} \\
\delta U= & \left(\frac{3}{2} \alpha-3 \beta\right) \frac{e^{2 U} r^{\prime 2}}{r^{2}}+\frac{1}{2} \alpha \frac{e^{2 U} r^{\prime} U^{\prime}}{r}-\beta e^{2 U} U^{\prime \prime} \\
& +y_{1} \frac{e^{2 U}}{r^{2}}+y_{2} \epsilon^{2 U} U^{\prime 2}+y_{3} \frac{e^{6 U}}{r^{4}} k^{2}
\end{aligned}
$$

where $x_{1}, x_{2}, x_{3}, y_{1}, y_{2}, y_{3}$ are six arbitrary parameters, which can be chosen at will to simplify the form of the final Lagrangian. After dropping a total derivative, the Lagrangian $\mathcal{L}$ becomes

$$
\begin{aligned}
\mathcal{L}= & 2+2 r^{\prime 2}-2 r^{2} U^{\prime 2}+\frac{e^{4 U}}{2 r^{2}} k^{2}+k \sigma^{\prime} \\
& +(-2 \alpha+4 \beta) \frac{e^{2 U}}{r^{2}}+\left(16 \alpha-24 \beta-4 x_{1}\right) \frac{e^{2 U} r^{\prime 2}}{r^{2}} \\
& +\left(-32 \alpha+48 \beta+8 x_{1}+8 y_{1}\right) \frac{e^{2 U} r^{\prime} U^{\prime}}{r}+\left(16 \alpha-24 \beta-4 x_{1}-8 y_{1}\right) e^{2 U} U^{\prime 2} \\
& +\left(-6 \alpha+28 \beta-\frac{4}{3} x_{2}\right) \frac{e^{2 U} r^{\prime 4}}{r^{2}}+\left(28 \alpha-\frac{152}{3} \beta+\frac{8}{3} x_{2}\right) \frac{e^{2 U} r^{\prime 3} U^{\prime}}{r} \\
& +\left(\frac{38}{3} \alpha-\frac{112}{3} \beta+\frac{16}{3} y_{2}\right) e^{2 U} r r^{\prime} U^{\prime 3} \\
& +\left(\frac{5}{3} \alpha+\frac{8}{3} \beta-\frac{8}{3} y_{2}\right) e^{2 U} r^{2} U^{\prime 4}+\left(-25 \alpha+60 \beta-4 x_{2}\right) e^{2 U} r^{\prime 2} U^{\prime 2} \\
& +\left(\frac{1}{2} \alpha+\frac{1}{4} \beta-x_{3}+2 y_{3}\right) k^{4} \frac{e^{10 U}}{r^{6}}+\left(2 \beta-x_{1}+2 y_{1}\right) \frac{e^{6 U}}{r^{4}} k^{2} \\
& +\left(\frac{33}{2} \alpha-32 \beta-x_{2}-12 x_{3}\right) \frac{e^{6 U} r^{\prime 2}}{r^{4}} k^{2}+\left(-25 \alpha+58 \beta+24 x_{3}+16 y_{3}\right) \frac{e^{6 U} r^{\prime} U^{\prime}}{r^{3}} k^{2} \\
& +\left(\frac{31}{4} \alpha-25 \beta-4 x_{3}+2 y_{2}-24 y_{3}\right) \frac{e^{6 U} U^{\prime 2}}{r^{2}} k^{2}
\end{aligned}
$$

This may be further simplified by reinstating the einbein $N$, and making use of the freedom to perform field redefinitions of $N$ preserving the property that the Lagrangian

\footnotetext{
${ }^{2}$ Additional first-order terms proportional to $e^{2 U} r^{\prime} / r$ and $e^{2 U} U^{\prime} / r$ would spoil one-dimensional diffeomorphism invariance and are therefore not considered. Moreover, we do not allow for field redefinitions of $k$, since $k$ corresponds to a conserved charge.
} 
only contains powers of first derivatives of fields:

$$
\delta N=t_{1} \frac{e^{2 U}}{r^{2}}+t_{2} \frac{e^{2 U} r^{2}}{r^{2}}+t_{3} e^{2 U} U^{\prime 2}+t_{4} \frac{e^{2 U} r^{\prime} U^{\prime}}{r}+t_{5} \frac{e^{6 U}}{r^{4}} k^{2}
$$

The Lagrangian becomes

$$
\begin{aligned}
\mathcal{L}= & 2+2 r^{\prime 2}-2 r^{2} U^{\prime 2}+\frac{e^{4 U}}{2 r^{2}} k^{2}+k \sigma^{\prime} \\
& +\left(-2 \alpha+4 \beta+2 t_{1}\right) \frac{e^{2 U}}{r^{2}}+\left(16 \alpha-24 \beta-4 x_{1}-2 t_{1}+2 t_{2}\right) \frac{e^{2 U} r^{\prime 2}}{r^{2}} \\
& +\left(-32 \alpha+48 \beta+8 x_{1}+8 y_{1}+2 t_{4}\right) \frac{e^{2 U} r^{\prime} U^{\prime}}{r}+\left(16 \alpha-24 \beta-4 x_{1}-8 y_{1}+2 t_{1}+2 t_{3}\right) e^{2 U} U^{\prime 2} \\
& +\left(-6 \alpha+28 \beta-\frac{4}{3} x_{2}-2 t_{2}\right) \frac{e^{2 U} r^{\prime 4}}{r^{2}}+\left(28 \alpha-\frac{152}{3} \beta+\frac{8}{3} x_{2}-2 t_{4}\right) \frac{e^{2 U} r^{\prime 3} U^{\prime}}{r} \\
& +\left(\frac{38}{3} \alpha-\frac{112}{3} \beta+\frac{16}{3} y_{2}+2 t_{4}\right) e^{2 U} r r^{\prime} U^{\prime 3} \\
& +\left(\frac{5}{3} \alpha+\frac{8}{3} \beta-\frac{8}{3} y_{2}+2 t_{3}\right) e^{2 U} r^{2} U^{\prime 4}+\left(-25 \alpha+60 \beta-4 x_{2}+2 t_{2}-2 t_{3}\right) e^{2 U} r^{\prime 2} U^{\prime 2} \\
& +\left(\frac{1}{2} \alpha+\frac{1}{4} \beta-x_{3}+2 y_{3}+\frac{1}{2} t_{5}\right) k^{4} \frac{e^{10 U}}{r^{6}}+\left(2 \beta-x_{1}+2 y_{1}+\frac{1}{2} t_{1}+2 t_{5}\right) \frac{e^{6 U}}{r^{4}} k^{2} \\
& +\left(\frac{33}{2} \alpha-32 \beta-x_{2}-12 x_{3}+\frac{1}{2} t_{2}-2 t_{5}\right) \frac{e^{6 U} r^{\prime 2}}{r^{4}} k^{2} \\
& +\left(-25 \alpha+58 \beta+24 x_{3}+16 y_{3}+\frac{1}{2} t_{4}\right) \frac{e^{6 U} r^{\prime} U^{\prime}}{r^{3}} k^{2} \\
& +\left(\frac{31}{4} \alpha-25 \beta-4 x_{3}+2 y_{2}-24 y_{3}+\frac{1}{2} t_{3}+2 t_{5}\right) \frac{e^{6 U} U^{\prime 2}}{r^{2}} k^{2}
\end{aligned}
$$

Remarkably, there exists a unique choice of the field redefinition ambiguities such that $\mathcal{L}$ reduces to its tree-level answer,

$$
\begin{gathered}
x_{1}=\frac{11}{4} \alpha-5 \beta, \quad x_{2}=-\frac{9}{4} \alpha+7 \beta, \quad x_{3}=\frac{1}{16}(17 \alpha-36 \beta) \\
y_{1}=-\frac{3}{2} \alpha+3 \beta, \quad y_{2}=-\frac{13}{2} \alpha+13 \beta, \quad y_{3}=\frac{1}{8}(-3 \alpha+2 \beta), \\
t_{1}=\alpha-2 \beta, \quad t_{2}=-\frac{3}{2}, \quad t_{3}=-\frac{19}{2} \alpha+16 \beta, \quad t_{4}=11 \alpha-16 \beta, \quad t_{5}=\frac{21}{8} \alpha-6 \beta
\end{gathered}
$$

We conclude that in the stationary, spherically symmetric sector, $R^{2}$ corrections to Einstein gravity can be completely eliminated by field redefinitions. In particular, the Ehlers symmetry is unbroken at this order. This result could have been anticipated [32] 
from the fact that, using field redefinitions of the four-dimensional graviton of the form $\delta g_{\mu \nu}=\kappa_{1} R_{\mu \nu}+\kappa_{2} g_{\mu \nu} R$, the $R^{2}$ corrections can always be related to the Gauss-Bonnet density, which is a total derivative. Clearly, higher order corrections such as $R^{4}$ cannot not be eliminated in the same fashion. Such terms have been discussed in [31].

\subsection{Einstein-Liouville Gravity}

We now consider Einstein gravity coupled to a scalar field $\phi$, and allow an arbitrary dependence of the $R^{2}$ couplings in $(2.1)$ on $\phi$,

$$
S=\int d^{4} x \sqrt{-g}\left[R_{4}+\frac{1}{2}(\partial \phi)^{2}+\alpha(\phi)\left(\left[R_{\mu \nu}^{(4)}\right]^{2}-\left[R^{(4)}\right]^{2}\right)+\beta(\phi)\left[R^{(4)}\right]^{2}+\gamma(\phi) R_{G B}^{2}\right]
$$

In particular, the term proportional to the Gauss-Bonnet density is no longer a total derivative, and we no longer expect to be able to remove all higher derivative corrections by field redefinitions. In the rest of this section we will refrain from displaying the $\phi$ dependence, and will indicate $\phi$-derivatives with a subscript, e.g. $\alpha_{\phi} \equiv d \alpha / d \phi$.

We now proceed as in section 2.1, by first performing field redefinitions and integrations by parts such that only powers of first derivatives appear in the Lagrangian. The most general field redefinition of $r$ and $U$ compatible with these requirements is again (2.11), while the field redefinition of $N$ must be generalized to

$$
\begin{aligned}
\delta N= & t_{1} \frac{e^{2 U}}{r^{2}}+t_{2} \frac{e^{2 U} r^{2}}{r^{2}}+t_{3} e^{2 U} U^{\prime 2}+t_{4} \frac{e^{2 U} r^{\prime} U^{\prime}}{r} \\
& +t_{5} \frac{e^{6 U}}{r^{4}} k^{2}+t_{6} e^{2 U} \phi^{\prime 2}+t_{7} e^{2 U} U^{\prime} \phi^{\prime}+t_{8} \frac{e^{2 U} r^{\prime} \phi^{\prime}}{r}
\end{aligned}
$$

and an extra field redefinition of $\phi$ must be introduced,

$$
\delta \phi=(5 \alpha-8 \beta) \frac{e^{2 U} r^{\prime} \phi^{\prime}}{r}+(-4 \alpha+8 \beta) e^{2 U} U^{\prime} \phi^{\prime}+z_{1} \frac{e^{2 U}}{r^{2}}+z_{2} e^{2 U} \phi^{2}+z_{3} \frac{e^{6 U}}{r^{4}} k^{2}
$$

Moreover, all coefficients $x_{i}, y_{i}, z_{i}, t_{i}$ may now depend on $\phi$. The full Lagrangian reads, after dropping a total derivative:

$$
\begin{aligned}
\mathcal{L}= & 2+\frac{1}{2} r^{2} \phi^{\prime 2}+2 r^{\prime 2}-2 r^{2} U^{\prime 2}+\frac{e^{4 U}}{2 r^{2}} k^{2}+k \sigma^{\prime} \\
& +\left(-2 \alpha+4 \beta+2 t_{1}\right) \frac{e^{2 U}}{r^{2}}+\left(16 \alpha-24 \beta+2 t_{1}+2 t_{3}-4 x_{1}-8 y_{1}\right) e^{2 U} U^{\prime 2}
\end{aligned}
$$




$$
\begin{aligned}
& +\left(32 \alpha+48 \beta+2 t_{4}+8 x_{1}+8 y_{1}\right) \frac{e^{2 U} r^{\prime} U^{\prime}}{r} \\
& +\left(16 \alpha-24 \beta-2 t_{1}+2 t_{2}-4 x_{1}\right) \frac{e^{2 U} r^{\prime 2}}{r^{2}}+\left(-\frac{1}{2} t_{1}+2 t_{6}+x_{1}+z_{1, \phi}\right) e^{2 U} \phi^{\prime 2} \\
& +\left(-12 \alpha_{\phi}+16 \beta_{\phi}+2 t_{8}+4 x_{1, \phi}+\frac{4}{3} x_{2, \phi}-2 z_{1}\right) \frac{e^{2 U} r^{\prime} \phi^{\prime}}{r} \\
& +\left(4 \alpha_{\phi}-8 \beta_{\phi}+8 \gamma_{\phi}+2 t_{7}-4 y_{1, \phi}+2 z_{1}\right) e^{2 U} U^{\prime} \phi^{\prime} \\
& +\left(\frac{5}{2} \alpha_{\phi}-4 \beta_{\phi}-\frac{1}{2} t_{8}-\frac{4}{3} z_{2}\right) e^{2 U} r^{\prime} \phi^{\prime 3}+\left(-2 \alpha_{\phi}+4 \beta_{\phi}-\frac{1}{2} t_{7}+\frac{2}{3} z_{2}\right) e^{2 U} U^{\prime} \phi^{\prime 3} \\
& +\left(-\frac{15}{2} \alpha+12 \beta-\frac{1}{2} t_{2}-2 t_{6}+x_{2}\right) e^{2 U} r^{\prime 2} \phi^{\prime 2}+\left(4 \alpha_{\phi}-16 \beta_{\phi}-2 t_{8}\right) \frac{e^{2 U} r^{\prime 3} \phi^{\prime}}{r} \\
& +\left(-\frac{15}{4} \alpha+7 \beta-\frac{1}{2} t_{3}+2 t_{6}\right) e^{2 U} r^{2} U^{\prime 2} \phi^{\prime 2} \\
& +\left(-25 \alpha+60 \beta+2 t_{2}-2 t_{3}-4 x_{2}\right) e^{2 U} r^{\prime 2} U^{\prime 2} \\
& +\left(-\alpha_{\phi}-4 \beta_{\phi}+16 \gamma_{\phi}+2 t_{8}\right) e^{2 U} r r^{\prime} U^{\prime 2} \phi^{\prime}+\left(8 \alpha-14 \beta-\frac{1}{2} t_{4}\right) e^{2 U} r r^{\prime} U^{\prime} \phi^{\prime 2} \\
& +\left(-10 \alpha_{\phi}+20 \beta_{\phi}-8 \gamma_{\phi}-2 t_{7}\right) e^{2 U} r^{\prime 2} U^{\prime} \phi^{\prime}+\left(\frac{38}{3} \alpha-\frac{112}{3} \beta+2 t_{4}+\frac{16}{3} y_{2}\right) e^{2 U} r r^{\prime} U^{\prime 3} \\
& +\left(\frac{4}{3} \alpha_{\phi}-\frac{8}{3} \beta_{\phi}-8 \gamma_{\phi}+2 t_{7}-\frac{4}{3} y_{2, \phi}\right) e^{2 U} U^{\prime 3} \phi^{\prime} \\
& +\left(28 \alpha-\frac{152}{3} \beta-2 t_{4}+\frac{8}{3} x_{2}\right) \frac{e^{2 U} r^{\prime 3} U^{\prime}}{r}+\left(-6 \alpha+\frac{28}{3} \beta-2 t_{2}-\frac{4}{3} x_{2}\right) \frac{e^{2 U} r^{\prime 4}}{r^{2}} \\
& +\left(-\frac{1}{2} t_{6}+\frac{1}{3} z_{2, \phi}\right) e^{2 U} r^{2} \phi^{\prime 4}+\left(\frac{5}{3} \alpha+\frac{8}{3} \beta+2 t_{3}-\frac{8}{3} y_{2}\right) e^{2 U} r^{2} U^{\prime 4} \\
& +\left(2 \beta+\frac{1}{2} t_{1}+2 t_{5}-x_{1}+2 y_{1}\right) \frac{e^{6 U}}{r^{4}} k^{2}+\left(-\frac{1}{2} t_{5}+\frac{1}{2} t_{6}+x_{3}+z_{3, \phi}\right) \frac{e^{6 U} \phi^{2}}{r^{2}} k^{2} \\
& +\left(\frac{31}{4} \alpha-25 \beta+\frac{1}{2} t_{3}+2 t_{5}-4 x_{3}+2 y_{2}-24 y_{3}\right) \frac{e^{6 U} U^{\prime 2}}{r^{2}} k^{2} \\
& +\left(-25 \alpha+58 \beta+\frac{1}{2} t_{4}+24 x_{3}+16 y_{3}\right) \frac{e^{6 U} r^{\prime} U^{\prime}}{r^{3}} k^{2} \\
& +\left(\frac{33}{2} \alpha-32 \beta+\frac{1}{2} t_{2}-2 t_{5}-x_{2}-12 x_{3}\right) \frac{e^{6 U} r^{2}}{r^{4}} k^{2} \\
& +\left(-\frac{9}{2} \alpha_{\phi}+8 \beta_{\phi}-4 \gamma_{\phi}+\frac{1}{2} t_{8}+4 x_{3, \phi}-4 z_{3}\right) \frac{e^{6 U} r^{\prime} \phi^{\prime}}{r^{3}} k^{2} \\
& +\left(\alpha_{\phi}-4 \beta_{\phi}+10 \gamma_{\phi}+\frac{1}{2} t_{7}-4 y_{3, \phi}+6 z_{3}\right) \frac{e^{6 U} U^{\prime} \phi^{\prime}}{r^{2}} k^{2} \\
& +\left(\frac{1}{2} \alpha+\frac{1}{4} \beta+\frac{1}{2} t_{5}-x_{3}+2 y_{3}\right) \frac{e^{10 U}}{r^{6}} k^{4}
\end{aligned}
$$


While there is no longer any choice of the coefficients $x_{i}, y_{i}, z_{i}, t_{i}$ which removes all higher derivative corrections, enforcing the same field redefinitions as in (2.15) (with all coefficients being now functions of $\phi$ ) ensures that all higher derivative contributions become proportional to $\phi^{\prime}$. This leaves six field redefinition ambiguities, which may be used e.g. to eliminate terms of cubic and quartic order in $U^{\prime}$ and $k$ :

$$
\begin{array}{rlrl}
z_{1}=-8 \gamma_{\phi} \quad, & z_{2}=-\frac{3}{4}\left(\alpha_{\phi}-2 \beta_{\phi}+2 \gamma_{\phi}\right), \\
z_{3}=-2 \gamma_{\phi} \quad, \quad t_{6}=-\frac{1}{2} \beta-z_{3, \phi}, \\
t_{7}=-5 \alpha_{\phi}+10 \beta_{\phi}+4 \gamma_{\phi} \quad, \quad t_{8}=\frac{1}{2} \alpha_{\phi}+2 \beta_{\phi}-8 \gamma_{\phi}
\end{array}
$$

Finally, we perform the Legendre transform over $k$, obtaining

$$
\begin{aligned}
L= & +\frac{1}{2} r^{2} \phi^{\prime 2}+2 r^{2}-2 r^{2} U^{\prime 2}-\frac{1}{2} e^{-4 U} r^{2} \sigma^{\prime 2} \\
& +\left(\frac{9}{4} \alpha-5 \beta-4 \gamma_{\phi \phi}\right) e^{2 U} \phi^{\prime 2}+\left(\frac{1}{4} \beta-\frac{1}{4} \alpha_{\phi \phi}+\frac{1}{2} \beta_{\phi \phi}\right) e^{2 U} r^{2} \phi^{\prime 4} \\
& +\left(\frac{13}{4} \alpha_{\phi}-7 \beta_{\phi}\right) e^{2 U} r r^{\prime} \phi^{\prime 3}+\left(-9 \alpha+20 \beta-4 \gamma_{\phi \phi}\right) e^{2 U} r^{\prime 2} \phi^{\prime 2}+16 \gamma_{\phi} \frac{e^{2 U} r^{\prime 3} \phi^{\prime}}{r} \\
& +\left(\frac{5}{2} \alpha-6 \beta\right) e^{2 U} r r^{\prime} U^{\prime} \phi^{\prime 2}-16 \gamma_{\phi} e^{2 U} r^{\prime 2} U^{\prime} \phi^{\prime}+\left(\alpha-2 \beta+4 \gamma_{\phi \phi}\right) e^{2 U} r^{2} U^{\prime 2} \phi^{\prime 2} \\
& +\left(-\frac{1}{4} \alpha+\frac{1}{2} \beta-\gamma_{\phi \phi}\right) e^{-2 U} r^{2} \phi^{\prime 2} \sigma^{\prime 2}
\end{aligned}
$$

In contrast to the pure gravity case, the higher-derivative terms now break the treelevel Ehlers symmetry (2.8) explicitly ${ }^{3}$. In section 3, we will show how the symmetry under a discrete subgroup of $S L(2, \mathbb{R})$ can be restored by including the contribution of Taub-NUT instantons.

\subsection{Einstein-Maxwell Gravity}

We now study the dimensional reduction of Einstein gravity coupled to an abelian gauge field $A$, in the presence of higher derivative corrections of the form:

$$
\begin{aligned}
S= & \int d^{4} x \sqrt{-g}\left[R_{4}-\frac{1}{4} F_{\mu \nu}^{2}+\alpha\left(\left[R_{\mu \nu}^{(4)}\right]^{2}-\left[R^{(4)}\right]^{2}\right)+\beta\left[R^{(4)}\right]^{2}\right. \\
& \left.+z_{1} F_{\mu \nu} F_{\nu \rho} F_{\rho \lambda} F_{\lambda \mu}+z_{2}\left(F_{\mu \nu}^{2}\right)^{2}+z_{3} \nabla^{\mu} F^{\nu \rho} \nabla_{\mu} F_{\nu \rho}+z_{4} R\left(F_{\mu \nu}\right)^{2}+o\left(\alpha^{\prime}\right)\right]
\end{aligned}
$$

\footnotetext{
${ }^{3}$ Strictly speaking, we cannot rule out that the S-matrix computed from the Lagrangian $(2.21)$ preserves Ehlers symmetry, although we find this possibility very unlikely.
} 
where $F_{\mu \nu}=\partial_{\mu} A_{\nu}-\partial_{\nu} A_{\mu}$. As before, we restrict to stationary spherically symmetric configurations of the form (2.4).

At two-derivative order, it is known that the reduced Lagrangian describes the geodesic motion of a fiducial particle on (a real cone over) the dimension 4 symmetric space $S U(2,1) / S l(2) \times U(1)[4,5,6]$. The four scalars correspond to the scalar $U$, the time component $\zeta$ of the Maxwell field $A_{4}$, the pseudo-scalar $\tilde{\zeta}$ dual to the reduced Maxwell field $A_{i}$, and the NUT potential $\sigma$ dual to the Kaluza-Klein connection $\omega_{i}$. Translations along the three axionic scalars $\zeta, \tilde{\zeta}, \sigma$ are generated by three conserved charges $q, p, k$ corresponding to the electric, magnetic and NUT charges, respectively; they satisfy an Heisenberg algebra $[p, q]=-2 k$, as a result of the non-trivial fibration of the $\sigma$ direction over the $(\zeta, \tilde{\zeta})$ plane. For simplicity, we shall restrict to static configurations with vanishing NUT charge, $k=0$. This allows us to express the electromagnetic field-strength directly in terms of the conserved charges $p, q$,

$$
F=p \sin \theta d \theta \wedge d \phi+i q \frac{e^{2 U} N}{r^{2}} d t \wedge d r
$$

The invariants are then

$$
\begin{gathered}
\sqrt{-g_{4}} F_{\mu \nu}^{2}=2 \frac{e^{2 U} N}{r^{2}}\left(p^{2}+q^{2}\right) \\
\sqrt{-g_{4}}\left(F_{\mu \nu}^{2}\right)^{2}=-2 \frac{e^{6 U} N}{r^{6}}\left(p^{4}+q^{4}\right) \\
\sqrt{-g_{4}} F_{\mu \nu} F_{\nu \rho} F_{\rho \lambda} F_{\lambda \mu}=4 \frac{e^{6 U} N}{r^{6}}\left(p^{2}+q^{2}\right)^{2} \\
\sqrt{-g_{4}} \nabla_{\mu} F_{\nu \rho} \nabla_{\mu} F_{\nu \rho}=12 \frac{e^{4 U}\left(r^{\prime}-r U^{\prime}\right)^{2}}{N r^{4}}\left(p^{2}+q^{2}\right)
\end{gathered}
$$

We now proceed as in Section 2.1, performing field redefinitions and integrations by parts so that only powers of $U^{\prime}$ and $r^{\prime}$ appear in the Lagrangian:

$$
\begin{aligned}
\delta r= & x_{1} \frac{e^{2 U}}{r}+x_{2} \frac{e^{2 U} r^{\prime 2}}{r}+x_{3} \frac{e^{4 U}}{r^{3}}\left(p^{2}+q^{2}\right)+\left(-\frac{5}{2} \alpha+4 \beta\right) e^{2 U} r^{\prime \prime} \\
& +(-\alpha+2 \beta) e^{2 U} r^{\prime} U^{\prime}+(2 \alpha-4 \beta) e^{2 U} r U^{\prime \prime}+\left(\frac{1}{4} \alpha-\beta\right) e^{2 U} r U^{\prime 2} \\
\delta U= & y_{1} \frac{e^{2 U}}{r^{2}}+y_{2} e^{2 U} U^{\prime 2}++y_{3} \frac{e^{4 U}}{r^{4}}\left(p^{2}+q^{2}\right)\left(\frac{3}{2} \alpha-3 \beta\right) \frac{e^{2 U} r^{\prime 2}}{r^{2}} \\
& +\frac{1}{2} \alpha \frac{e^{2 U} r^{\prime} U^{\prime}}{r}-\beta e^{2 U} U^{\prime \prime} \\
\delta N= & t_{1} \frac{e^{2 U}}{r^{2}}+t_{2} \frac{e^{2 U} r^{\prime 2}}{r^{2}}+t_{3} e^{2 U} U^{\prime 2}+t_{4} \frac{e^{2 U} r^{\prime} U^{\prime}}{r}+t_{5} \frac{e^{4 U}}{r^{4}}\left(p^{2}+q^{2}\right)
\end{aligned}
$$


After dropping total derivatives the Lagrangian becomes

$$
\begin{aligned}
\mathcal{L}= & +2 r^{\prime 2}-2 r^{2} U^{\prime 2}-\frac{e^{2 U}}{2 r^{2}}\left(p^{2}+q^{2}\right)+\left(-2 \alpha+4 \beta+2 t_{1}\right) \frac{e^{2 U}}{r^{2}} \\
& +\left(16 \alpha-24 \beta-4 x_{1}-2 t_{1}+2 t_{2}\right) \frac{e^{2 U} r^{\prime 2}}{r^{2}}+\left(-32 \alpha+48 \beta+8 x_{1}+8 y_{1}+2 t_{4}\right) \frac{e^{2 U} r^{\prime} U^{\prime}}{r} \\
& +\left(16 \alpha-24 \beta-4 x_{1}-8 y_{1}+2 t_{1}+2 t_{3}\right) e^{2 U} U^{\prime 2} \\
& +\left(-6 \alpha+\frac{28}{3} \beta-\frac{4}{3} x_{2}-2 t_{2}\right) \frac{e^{2 U} r^{\prime 4}}{r^{2}}+\left(28 \alpha-\frac{152}{3} \beta+\frac{8}{3} x_{2}-2 t_{4}\right) \frac{e^{2 U} r^{\prime 3} U^{\prime}}{r} \\
& +\left(\frac{38}{3} \alpha-\frac{112}{3} \beta+\frac{16}{3} y_{2}+2 t_{4}\right) e^{2 U} r r^{\prime} U^{\prime 3} \\
& +\left(\frac{5}{3} \alpha+\frac{8}{3} \beta-\frac{8}{3} y_{2}+2 t_{3}\right) e^{2 U} r^{2} U^{\prime 4}+\left(-25 \alpha+60 \beta-4 x_{2}+2 t_{2}-2 t_{3}\right) e^{2 U} r^{\prime 2} U^{\prime 2} \\
& +\left(-\frac{1}{2} t_{1}+2 t_{5}+x_{1}-y_{1}+4 z_{4}\right) e^{4 U} r^{4}\left(p^{2}+q^{2}\right) \\
& +\left(-\frac{1}{2} t_{2}-2 t_{5}+x_{2}-12 x_{3}+12 z_{3}-28 z_{4}\right) \frac{e^{4 U} r^{\prime 2}}{r^{4}}\left(p^{2}+q^{2}\right) \\
& +\left(-\frac{1}{2} t_{4}+16 x_{3}+16 y_{3}-24 z_{3}+48 z_{4}\right) \frac{e^{4 U} r^{\prime} U^{\prime}}{r^{3}}\left(p^{2}+q^{2}\right) \\
& +\left(-\frac{1}{2} t_{3}+2 t_{5}-4 x_{3}-y_{2}-16 y_{3}+12 z_{3}-20 z_{4}\right) \frac{e^{4 U} U^{\prime 2}}{r^{2}}\left(p^{2}+q^{2}\right) \\
& +\left(-\frac{1}{2} t_{5}+x_{3}-y_{3}+4 z_{1}\right) \frac{e^{6 U}}{r^{6}}\left(p^{2}+q^{2}\right)^{2}-2 z_{2} \frac{e^{6 U}}{r^{6}}\left(p^{4}+q^{4}\right) \\
& +(-2)
\end{aligned}
$$

Contrary to the pure gravity case, it is no longer possible to cancel the higher-derivative corrections by appropriate choices of the field redefinition ambiguities. A convenient choice is to set

$$
\begin{gathered}
x_{1}=\frac{9}{8} \alpha-\frac{2}{5}\left(7 \beta+8 z_{1}+z_{3}\right), \quad x_{2}=-\frac{9}{4} \alpha+7 \beta \\
x_{3}=-\frac{7}{32} \alpha+\frac{11}{20} \beta-\frac{4}{5} z_{1}+\frac{9}{10} z_{3}-2 z_{4} \\
y_{1}=\frac{1}{40}\left(5 \alpha+32 \beta+128 z_{1}+16 z_{3}\right), \quad y_{2}=-\frac{13}{2} \alpha+13 \beta \\
y_{3}=-\frac{1}{20}\left(10 \alpha-9 \beta-16 z_{1}+40 z_{2}-12 z_{3}+20 z_{4},\right. \\
t_{1}=\frac{1}{20}\left(85 \alpha+16\left(-8 \beta+8 z_{1}+z_{3}\right)\right), \quad t_{2}=-\frac{3}{2} \alpha, \quad t_{3}=-\frac{19}{2} \alpha+16, \\
t_{4}=11 \alpha-16 \beta, \quad t_{5}=\frac{1}{80}\left(45 \alpha+16 \beta+384 z_{1}+48 z_{3}-160 z_{4}\right)
\end{gathered}
$$


leading to the Lagrangian

$$
\begin{aligned}
\mathcal{L}= & 2+2 r^{\prime 2}-2 r^{2} U^{\prime 2}-\frac{e^{2 U}}{2 r^{2}}\left(p^{2}+q^{2}\right) \\
& +\left(\frac{13}{2} \alpha-\frac{44}{5} \beta+\frac{64}{5} z_{1}+\frac{8}{5} z_{3}\right) \frac{e^{2 U}}{r^{2}}+4 z_{2} \frac{e^{6 U}}{r^{6}} p^{2} q^{2} \\
& +\left(-17 \alpha+24 \beta-32 z_{2}\right) \frac{e^{4 U} r^{\prime} U^{\prime}}{r^{3}}\left(p^{2}+q^{2}\right) \\
& +\left(\frac{85}{4} \alpha-30 \beta+32 z_{2}\right) \frac{e^{4 U} U^{\prime 2}}{r^{2}}\left(p^{2}+q^{2}\right)
\end{aligned}
$$

This makes it clear that higher-derivative corrections can be eliminated only when the five couplings in the bare Lagrangian satisfy the three relations

$$
\alpha=\frac{24}{17} \beta, \quad z_{2}=0, \quad 8 z_{1}+z_{3}=-\alpha / 6
$$

It is of interest to study whether the Lagrangian (2.29) admits solutions with flat spatial slices, as is necessary for the existence of extremal black holes. To answer this question, one must check whether the choices $N=1 / \rho^{2}$ and $r=1 / \rho$ are consistent with the equations of motion of $N$ and $r$. Computation shows that this is case only when $z_{2}=0$. This is in fact part of the rationale for the choice (2.30), since, for general field redefinition ambiguities, the conditions for the compatibility of flat slices are given by

$$
\begin{aligned}
z_{2} & =0 \\
x_{1} & =\frac{1}{40}\left(-265 \alpha+488 \beta+20 t_{4}-40 x_{2}-128 z_{1}-16 z_{3}\right) \\
x_{3} & =-\frac{7}{32} \alpha+\frac{11}{20} \beta-\frac{4}{5} z_{1}+\frac{9}{10} z_{3}-2 z_{4} \\
t_{1} & =\frac{33}{4} \alpha+\frac{1}{10}\left(-144 \beta-10 t_{2}-5 t_{4}+64 z_{1}+8 z_{3}\right) \\
t_{3} & =-\frac{29}{4} \alpha+\frac{4}{5}\left(21 \beta-5 t_{5}+24 z_{1}+3 z_{3}-10 z_{4}\right)
\end{aligned}
$$

satisfied by (2.30). Thus, we find that the assumption of the existence of extremal black holes (more specifically, the consistency of the reduction to flat spatial slices) requires that there should be no $\left(F^{2}\right)^{2}$ term in the Lagrangian. It would be interesting to relate this condition to the possibility of supersymmetrizing the Lagrangian (2.22).

\section{Ehlers Symmetry Restored}

One of the main results of the previous Section is that there exists a choice of field redef-

initions such that the one-dimensional Lagrangian describing four-dimensional gravity 
in the stationary, spherically symmetric sector involves only powers of first derivatives. While this choice ensures the absence of spurious modes and at the same makes the canonical quantization straightforward, it is also a particularly convenient frame to discuss the invariance under the Ehlers symmetry $\operatorname{Sl}(2, \mathbb{R})$.

Indeed, returning to the Lagrangian (2.19) for Einstein-Liouville gravity with general spatial slices, we may perform the Legendre transform ${ }^{4}$ over the NUT charge $k$, so as to express the result as a function of $U^{\prime}$ and $\sigma^{\prime}$. It is useful to change basis to

$$
p=2 i U^{\prime}+e^{-2 U} \sigma^{\prime}, \quad \bar{p}=-2 i U^{\prime}+e^{-2 U} \sigma^{\prime},
$$

where $p=(d \tau / d \rho) / \tau_{2}$ is (the pull back of) the left-invariant one-form on $S l(2, \mathbb{R}) / U(1)$, transforming by a phase under the action $(2.8)$ of $S l(2, \mathbb{R})$,

$$
p \rightarrow\left(\frac{c \bar{\tau}+d}{c \tau+d}\right) p
$$

This leads to the Lagrangian

$$
\begin{aligned}
L= & 2+2 r^{\prime 2}+\frac{1}{2} r^{2} \phi^{\prime 2}-\frac{1}{2} r^{2} p \bar{p} \\
& +\left(\frac{9}{4} \alpha-5 \beta-4 \gamma_{\phi \phi}\right) e^{2 U} \phi^{\prime 2}+\frac{1}{4} \beta e^{2 U} r^{2} \phi^{\prime 4} \\
& +\left(\frac{13}{4} \alpha_{\phi}-7 \beta_{\phi}\right) e^{2 U} r r^{\prime} \phi^{\prime 3}+\left(-9 \alpha+20 \beta-4 \gamma_{\phi \phi}\right) e^{2 U} r^{\prime 2} \phi^{\prime 2} \\
& +16 \gamma_{\phi} \frac{e^{2 U} r^{\prime 3} \phi^{\prime}}{r}+\left(-\frac{1}{4} \alpha_{\phi \phi}+\frac{1}{2} \beta_{\phi \phi}\right) e^{2 U} r^{2} \phi^{\prime 4} \\
& +i\left(-\frac{5}{8} \alpha+\frac{3}{2} \beta\right) e^{2 U} r r^{\prime} \phi^{\prime 2}(p-\bar{p})+4 i \gamma_{\phi} e^{2 U} r^{\prime 2} \phi^{\prime}(p-\bar{p}) \\
& +\left(-\frac{1}{8} \alpha+\frac{1}{4} \beta-\frac{1}{2} \gamma_{\phi \phi}\right) e^{2 U} r^{2} \phi^{\prime 2}\left(p^{2}+\bar{p}^{2}\right)
\end{aligned}
$$

This Lagrangian can be made invariant under a discrete $S l(2, \mathbb{Z})$ subgroup of the Ehlers symmetry provided any term $a_{m, n} e^{2 U} p^{m} \bar{p}^{n}$ is replaced by $f_{1, m-n}(\tau, \bar{\tau}) p^{m} \bar{p}^{n}$, where $f_{1, m-n}$ is a modular form of modular weight $0, U(1)$ charge $m-n$ behaving as $f_{m-n} \sim a_{m, n} e^{2 U}$ in the limit where $U \rightarrow \infty$. Such modular forms have already made an appearance in the physics literature in discussions of the S-duality invariance of the type IIB string in ten dimensions $[25,26]$ and can be expressed as generalized non-holomorphic Eisenstein series

$$
f_{s, k}(\tau, \bar{\tau})=\sum_{(p, q) \neq(0,0)} \frac{\tau_{2}^{s}}{(p \tau+q)^{s+k}(p \bar{\tau}+q)^{s-k}}
$$

\footnotetext{
${ }^{4}$ Consistently with our perturbative analysis, one should retain only the branch where $\sigma^{\prime}=$ $-k e^{-4 U} / r^{2}+\mathcal{O}\left(\alpha^{\prime}\right)$.
} 
which satisfy $\bar{f}_{s, k}=f_{s,-k}$. Under modular transformations the functions $f_{s, k}(\tau, \bar{\tau})$ transform as:

$$
f_{s, k}(\tau, \bar{\tau}) \rightarrow\left(\frac{c \tau+d}{c \bar{\tau}+d}\right)^{k} f_{s, k}(\tau, \bar{\tau})
$$

The leading behaviour as $U \rightarrow \infty$ uniquely selects $s=1$. Using the identity

$$
\left(k+2 i \tau_{2} \partial_{\tau}\right) f_{s, k}=(s+k) f_{k+1}
$$

and the known expression for $f_{1,0}$,

$$
f_{1,0}=-\pi \log \left(\tau_{2}|\eta(\tau)|^{4}\right)
$$

it is easy to express $f_{s=1, k}$ for relevant values ${ }^{5}$ of $k$ in terms of the standard holomorphic and almost holomorphic modular forms $E_{4}, E_{6}$ and $\hat{E}_{2}=E_{2}-\frac{3}{\pi \tau_{2}}$ :

$$
\begin{gathered}
f_{1,1}=\frac{\pi^{2} \tau_{2}}{3} \hat{E}_{2}, \quad f_{1,2}=\frac{\pi^{3} \tau_{2}^{2}}{18}\left(E_{4}-\hat{E}_{2}^{2}\right) \\
f_{1,3}=\frac{\pi^{4} \tau_{2}^{3}}{81}\left(2 E_{6}-3 \hat{E}_{2} E_{4}+\hat{E}_{2}^{3}\right), \quad f_{1,4}=\frac{\pi^{5} \tau_{2}^{4}}{324}\left(3 E_{4}^{2}-8 \hat{E}_{2} E_{6}+6 \hat{E}_{2}^{2} E_{4}-\hat{E}_{2}^{4}\right)
\end{gathered}
$$

The large radius $\tau_{2} \rightarrow \infty$ expansion for these functions reads:

$$
f_{1, k}=\frac{\pi^{2}}{3} \tau_{2}-\frac{\pi}{k}+\mathcal{O}\left(e^{-\tau_{2}}\right)
$$

which also applies to $k=0$ upon replacing $1 / k$ by $\log \tau_{2}$. The Lagrangian (3.3) can then be covariantized under $S l(2, \mathbb{Z})$ into

$$
\begin{aligned}
L_{c o v}= & +2 r^{\prime 2}+\frac{1}{2} r^{2} \phi^{\prime 2}-\frac{1}{2} r^{2} p \bar{p} \\
& +\left(\frac{9}{4} \alpha-5 \beta-4 \gamma_{\phi \phi}\right) \tilde{f}_{(1,0)} \phi^{\prime 2}+\frac{1}{4} \beta \tilde{f}_{(1,0)} r^{2} \phi^{\prime 4} \\
& +\left(\frac{13}{4} \alpha_{\phi}-7 \beta_{\phi}\right) \tilde{f}_{(1,0)} r r^{\prime} \phi^{\prime 3}+\left(-9 \alpha+20 \beta-4 \gamma_{\phi \phi}\right) \tilde{f}_{(1,0)} r^{\prime 2} \phi^{\prime 2} \\
& +16 \gamma^{\prime} \tilde{f}_{(1,0)} \frac{r^{\prime 3} \phi^{\prime}}{r}+\left(-\frac{1}{4} \alpha_{\phi \phi}+\frac{1}{2} \beta_{\phi \phi}\right) \tilde{f}_{(1,0)} r^{2} \phi^{\prime 4} \\
& +i\left(-\frac{5}{8} \alpha+\frac{3}{2} \beta\right) r r^{\prime} \phi^{\prime 2}\left(\tilde{f}_{(1,1)} p-\tilde{f}_{(1,1-)} \bar{p}\right)+4 i \gamma^{\prime} r^{\prime 2} \phi^{\prime}\left(\tilde{f}_{(1,1)} p-\tilde{f}_{(1,-1)} \bar{p}\right) \\
& +\left(-\frac{1}{8} \alpha+\frac{1}{4} \beta-\frac{1}{2} \gamma_{\phi \phi}\right) r^{2} \phi^{\prime 2}\left(\tilde{f}_{(1,2)} p^{2}+\tilde{f}_{(1,-2)} \bar{p}^{2}\right)
\end{aligned}
$$

\footnotetext{
${ }^{5}$ The Eisenstein series $f_{1,3}$ and $f_{1,4}$ would become useful if we chose not to eliminate the cubic and quartic terms in $U^{\prime}$ and $k$ in (2.19).
} 
with $\frac{\pi^{2}}{3} \tilde{f}_{s, k}=f_{s, k}$. It agrees with the Lagrangian (3.3) from dimensional reduction in the limit $\tau_{2}=e^{2 U} \rightarrow \infty$, but differs by perturbative terms of order $1 / \tau_{2}$ and an infinite series of exponentially suppressed terms of order $e^{-m \tau_{2} \pm i m \tau_{1}}$ The former can be attributed to loops of gravitons running around the compact circle, while the latter can be naturally attributed to Taub-NUT instantons ${ }^{6}$ : the classical action of these gravitational self-dual instantons, with topology $\mathbb{R}^{3} \times S^{1}$ at infinity, scales as the square of the radius of the compact direction $e^{2 U}=\tau_{2}$ in Planck units, and includes a linear coupling to the NUT scalar $\sigma=\tau_{1}$ proportional to the NUT charge.

Thus, we have given a precise realization of the proposal outlined in [23], in the toy-model of Einstein-Liouville gravity compactified on a circle. It is clear that this procedure works irrespective of the details of the higher-derivative action, provided one has managed to express it in powers of first derivatives of $U$ and $\sigma$ only (the cone variable $r$ is spectator in this discussion). It would be interesting to study the fate of the $S U(2,1)$ symmetry of the Einstein-Maxwell theory (or its extension to EinsteinMaxwell-Liouville theories) along similar lines.

\section{C-map with Higher Derivative Corrections}

In this section we give a preliminary discussion of the relation between the higher derivative amplitudes $F_{1}$ and $\tilde{F}_{1}$ on the vector and hypermultiplet branch, focussing on the simplest case of a single universal multiplet, $n_{H}=1$, and no vector multiplet $n_{V}=0$ in four dimensions. After dimensional reduction to 3 dimensions, the moduli space consists of two copies of the quaternionic-Kähler space $S U(2,1) / S U(2) \times U(1)$, associated to $U, \zeta, \tilde{\zeta}, \sigma$ on the vector side, and $\varphi, \chi, \tilde{\chi}, a$ on the hypermultiplet side, where $S=a+i e^{-2 \varphi}$ and $Z=\chi+i \tilde{\chi}$ are the two chiral multiplets in the universal hypermultiplet, $\varphi$ being the four dimensional dilaton. For simplicity, we shall also restrict to the $S l(2) / U(1)$ sector of these two moduli spaces, retaining only $(U, \sigma)$ and $(\varphi, a)$. According to $[27,30]$, the hypermultiplet branch in four dimensions may receive higher-derivative corrections of the form

$$
S_{H}=\int d^{4} x \sqrt{-g_{4}}\left[\frac{\nabla_{\mu} S \nabla^{\mu} \bar{S}}{S_{2}^{2}}+\tilde{\alpha} \frac{\nabla_{\mu \nu} S \nabla^{\mu \nu} S+\nabla_{\mu \nu} \bar{S} \nabla^{\mu \nu} \bar{S}}{S_{2}^{2}}\right]
$$

where $\tilde{\alpha}$ is in general a function of all hypermultiplets, receiving a one-loop contribution plus instanton corrections. In line with our simplifying assumptions, and consistently with the fact that it arises at one-loop, we shall assume that $\tilde{\alpha}$ is just a constant.

\footnotetext{
${ }^{6}$ Contributions of Taub-NUT instantons to three-dimensional string theories have been analyzed in $[29]$.
} 
Moreover, we assume that the $R^{2}$ couplings in four dimensions are given by (2.1) where $\alpha$ is also a constant, corresponding to a one-loop contribution in string theory, and $\beta$ is set to zero.

Upon reducing on a stationary, spherically symmetric metric with flat spatial slices,

$$
d s^{2}=-e^{2 U}(d t+k \cos \theta d \varphi)^{2}+e^{-2 U}\left(\frac{d \rho^{2}}{\rho^{4}}+\frac{1}{\rho^{2}} d \Omega_{2}^{2}\right)
$$

and performing the now standard field redefinitions to eliminate powers of $\varphi^{\prime \prime}$ and $a^{\prime \prime}$, the combined four-dimensional action (2.1) plus (4.1) reduces to a one-dimensional Lagrangian of the form

$$
\begin{aligned}
\mathcal{L}= & -2 U^{\prime 2}-\frac{1}{2} e^{-4 U} \sigma^{\prime 2}-2 \varphi^{\prime 2}-\frac{1}{2} e^{4 \varphi} a^{\prime 2} \\
& +\alpha e^{2 U} \rho^{4}\left(U^{\prime 4}+e^{-8 U} \sigma^{\prime 4}\right)+\tilde{\alpha} e^{2 U} \rho^{4}\left(\varphi^{\prime 4}+e^{8 \varphi} a^{\prime 4}\right)
\end{aligned}
$$

where $k$ was dualized into $\sigma^{\prime}$. In writing (4.3), we have not paid attention to the detailed form of the higher-derivative interactions, but only exhibited their exponential dependance on $U$ and $\varphi$. At tree-level, $\mathcal{L}$ is invariant under

$$
U \rightarrow-\varphi, \quad \varphi \rightarrow-U, \quad \sigma \leftrightarrow a
$$

In type II string theory, this symmetry is realized by T-duality along the fourth circle, which exchanges the vector and hypermultiplet branches in three dimensions. The point to be emphasized now is that the symmetry (4.4) is broken by the higher-derivative interactions in (4.3), unless both $\alpha$ and $\tilde{\alpha}$ vanish. Indeed, a constant $\alpha$ would imply that $\tilde{\alpha} \sim e^{-2 \varphi-2 U}$, which would correspond to a tree-level $\left(\nabla^{2} S\right)^{2}$ contribution which vanishes in the decompactification limit $U \rightarrow \infty$. Similarly, a constant $\tilde{\alpha}$ would imply that $\alpha \sim e^{-2 \varphi-2 U}$, again a tree-level $R^{2}$ correction vanishing in the decompactification limit. Either of these options would be disastrous. Moreover, a puzzling feature of (4.3) is that the vector and hypermultiplet branches are not decoupled, in contrary to common belief.

These conundrums can be simply avoided by noting that, as shown in Section 2.1 , there exists a field redefinition scheme which removes any moduli-independent $R^{2}$ correction. Similarly, there should exist field redefinitions on the hypermultiplet side which allow to remove any moduli-independent $\left(\nabla^{2} S\right)^{2}$ coupling. While it is not the goal of this paper to perform a systematic analysis of this problem, we feel that the simple observation above should serve as a word of caution when trying to understand the relation between topological amplitudes on the vector and hypermultiplet side. 


\section{Acknowledgments}

The authors are grateful to N. Lambert and P. West for useful correspondence. We also thank the authors of [32] for pointing out a trivial claim in an earlier version of this work. The research of B.P. is supported by the EU under contracts MTRNCT-2004-005104, MTRN-CT-2004-512194, and by ANR (CNRS-USAR) contract No 05-BLAN-0079-01.

\section{References}

[1] P. Breitenlohner, D. Maison and G. W. Gibbons, "Four-Dimensional Black Holes from Kaluza-Klein Theories," Commun. Math. Phys. 120 (1988) 295.

[2] R. Geroch, "A Method for generating solutions of Einstein's equations," J. Math. Phys. 12, 918 (1971).

[3] J. Ehlers, in "Les Théories Physiques de la Gravitation", CNRS, Paris (1959);

[4] W. Kinnersley, "Symmetries Of The Stationary Einstein-Maxwell Field Equations. I," J. Math. Phys. 18 (1977) 1529.

[5] P. O. Mazur, "Black Hole Uniqueness From A Hidden Symmetry Of Einstein's Gravity," Gen. Rel. Grav. 16 (1984) 211.

[6] B. Pioline, "Lectures on on black holes, topological strings and quantum attractors," Class. Quant. Grav. 23 (2006) S981 [arXiv:hep-th/0607227].

[7] P. Breitenlohner and D. Maison, "On nonlinear sigma-models arising in (super-)gravity," Commun. Math. Phys. 209 (2000) 785 [arXiv:gr-qc/9806002].

[8] M. Gunaydin, S. McReynolds and M. Zagermann, "Unified N = 2 Maxwell-Einstein and Yang-Mills-Einstein supergravity theories in four dimensions," JHEP 0509 (2005) 026 [arXiv:hep-th/0507227].

[9] M. Gunaydin, G. Sierra and P. K. Townsend, "The Geometry Of N=2 Maxwell-Einstein Supergravity And Jordan Algebras," Nucl. Phys. B 242 (1984) 244.

[10] S. Cecotti, S. Ferrara and L. Girardello, Int. J. Mod. Phys. A 4 (1989) 2475.

[11] S. Ferrara and S. Sabharwal, "Quaternionic Manifolds for Type II Superstring Vacua of Calabi-Yau Spaces," Nucl. Phys. B 332 (1990) 317.

[12] M. Gunaydin, A. Neitzke, B. Pioline and A. Waldron, "BPS black holes, quantum attractor flows and automorphic forms," Phys. Rev. D 73 (2006) 084019 [arXiv:hep-th/0512296]; "Quantum Attractor Flows", to appear. 
[13] A. Neitzke, B. Pioline and S. Vandoren, "Twistors and black holes," JHEP 0704, 038 (2007) [arXiv:hep-th/0701214].

[14] A. Dabholkar, "Exact counting of black hole microstates," Phys. Rev. Lett. 94, 241301 (2005) [arXiv:hep-th/0409148].

[15] A. Dabholkar, R. Kallosh and A. Maloney, "A stringy cloak for a classical singularity," JHEP 0412, 059 (2004) [arXiv:hep-th/0410076].

[16] A. Sen, "How does a fundamental string stretch its horizon?," JHEP 0505 (2005) 059 [arXiv:hep-th/0411255].

[17] V. Hubeny, A. Maloney and M. Rangamani, "String-corrected black holes," JHEP 0505 (2005) 035 [arXiv:hep-th/0411272].

[18] A. Dabholkar, F. Denef, G. W. Moore and B. Pioline, "Exact and asymptotic degeneracies of small black holes," JHEP 0508, 021 (2005) [arXiv:hep-th/0502157].

[19] A. Dabholkar, F. Denef, G. W. Moore and B. Pioline, "Precision counting of small black holes," JHEP 0510, 096 (2005) [arXiv:hep-th/0507014].

[20] B. Zwiebach, "Curvature Squared Terms And String Theories," Phys. Lett. B 156 (1985) 315.

[21] K. A. Meissner, "Symmetries of higher-order string gravity actions," Phys. Lett. B 392 (1997) 298 [arXiv:hep-th/9610131].

[22] N. Kaloper and K. A. Meissner, "Duality beyond the first loop," Phys. Rev. D 56 (1997) 7940 [arXiv:hep-th/9705193].

[23] N. Lambert and P. West, "Enhanced coset symmetries and higher derivative corrections," Phys. Rev. D 74 (2006) 065002 [arXiv:hep-th/0603255].

[24] N. Lambert and P. West, "Duality groups, automorphic forms and higher derivative corrections," Phys. Rev. D 75 (2007) 066002 [arXiv:hep-th/0611318].

[25] M. B. Green, M. Gutperle and H. h. Kwon, "lambda**16 and related terms in M-theory on T**2," Phys. Lett. B 421, 149 (1998) [arXiv:hep-th/9710151].

[26] A. Kehagias and H. Partouche, "D-instanton corrections as (p,q)-string effects and non-renormalization theorems," Int. J. Mod. Phys. A 13 (1998) 5075 [arXiv:hep-th/9712164].

[27] I. Antoniadis, E. Gava, K. S. Narain and T. R. Taylor, "Topological amplitudes in string theory," Nucl. Phys. B 413 (1994) 162 [arXiv:hep-th/9307158]. 
[28] S. W. Hawking and T. Hertog, "Living with ghosts," Phys. Rev. D 65 (2002) 103515 [arXiv:hep-th/0107088].

[29] N. A. Obers and B. Pioline, "Exact thresholds and instanton effects in D $=3$ string theories," JHEP 0007 (2000) 003 [arXiv:hep-th/0006088].

[30] I. Antoniadis, B. Pioline and T. R. Taylor, "Calculable e**(-1/lambda) effects," Nucl. Phys. B 512 (1998) 61 [arXiv:hep-th/9707222].

[31] L. Bao, M. Cederwall and B. E. W. Nilsson, "Aspects of higher curvature terms and U-duality," arXiv:0706.1183 [hep-th].

[32] C. Colonnello and A. Kleinschmidt, "Ehlers symmetry at the next derivative order," arXiv:0706.2816 [hep-th]. 